\title{
Actively-targeted LTVSPWY peptide-modified magnetic nanoparticles for tumor imaging
}

This article was published in the following Dove Press journal:

International Journal of Nanomedicine

23 July 2012

Number of times this article has been viewed

\section{Li-Yong Jie' \\ Li-Li Cai ${ }^{2}$ \\ Le-Jian Wang ${ }^{2}$ \\ Xiao-Ying Ying ${ }^{2}$ \\ Ri-Sheng $Y^{\prime}{ }^{\prime}$ \\ Min-Ming Zhang' \\ Yong-Zhong $\mathrm{Du}^{2}$}

'Department of Radiology, The Second Affiliated Hospital, Zhejiang University School of Medicine, ${ }^{2}$ College of Pharmaceutical Sciences, Zhejiang University, Hangzhou, People's Republic of China
Correspondence: Yong-Zhong Du College of Pharmaceutical Sciences, Zhejiang University, 866 Yuhangtang Road, Hangzhou 310058, People's Republic of China Tel $+8657 \mid 88208439$

Fax +86 57। 88208439

Email duyongzhong@zju.edu.cn

Min-Ming Zhang Department of Radiology, The Second Affiliated Hospital, Zhejiang University School of Medicine, Hangzhou 310009 , China

$\mathrm{Tel} / \mathrm{Fax}+86$ 57| 873I5255

Email cjr.zhangminming@vip.163.com
Background: Magnetic resonance imaging (MRI) is widely used in modern clinical medicine as a diagnostic tool, and provides noninvasive and three-dimensional visualization of biological phenomena in living organisms with high spatial and temporal resolution. Therefore, considerable attention has been paid to magnetic nanoparticles as MRI contrast agents with efficient targeting ability and cellular internalization ability, which make it possible to offer higher contrast and information-rich images for detection of disease.

Methods: LTVSPWY peptide-modified PEGylated chitosan (LTVSPWY-PEG-CS) was synthesized by chemical reaction, and the chemical structure was confirmed by ${ }^{1} \mathrm{H}-\mathrm{NMR}$. LTVSPWY-PEG-CS-modified magnetic nanoparticles were prepared successfully using the solvent diffusion method. Their particle size, size distribution, and zeta potential were measured by dynamic light scattering and electrophoretic mobility, and their surface morphology was investigated by transmission electron microscopy. To investigate their selective targeting ability, the cellular uptake of the LTVSPWY-PEG-CS-modified magnetic nanoparticles was observed in a cocultured system of SKOV-3 cells which overexpress HER2 and A549 cells which are HER2-negative. The in vitro cytotoxicity of these nanoparticles in SKOV-3 and A549 cells was measured using the MTT method. The SKOV-3-bearing nude mouse model was used to investigate the tumor targeting ability of the magnetic nanoparticles in vivo.

Results: The average diameter and zeta potential of the LTVSPWY-PEG-CS-modified magnetic nanoparticles was $267.3 \pm 23.4 \mathrm{~nm}$ and $30.5 \pm 7.0 \mathrm{mV}$, respectively, with a narrow size distribution and spherical morphology. In vitro cytotoxicity tests demonstrated that these magnetic nanoparticles were carriers suitable for use in cancer diagnostics with low toxicity. With modification of the LTVSPWY homing peptide, magnetic nanoparticles could be selectively taken up by SKOV-3 cells overexpressing HER2 when cocultured with HER2-negative A549 cells. In vivo biodistribution results suggest that treatment with LTVSPWY-PEG-CSmodified magnetic nanoparticles/DiR enabled tumors to be identified and diagnosed more rapidly and efficiently in vivo.

Conclusion: LTVSPWY-PEG-CS-modified magnetic nanoparticles are a promising contrast agent for early detection of tumors overexpressing HER2 and further diagnostic application.

Keywords: LTVSPWY peptide, HER2, poly(ethylene glycol), chitosan, magnetic nanoparticles, tumor targeting

\section{Introduction}

During recent decades, use of magnetic nanoparticles in biomedical applications, such as magnetic drug delivery, magnetic resonance imaging (MRI), and cell and tissue targeting, has drawn considerable attention due to their unique magnetic properties and relatively small size as biological entities. ${ }^{1}$ The responsiveness of magnetic 
nanoparticles to external magnetic fields is fully exploited when they are used as drug delivery systems, whereby chemotherapeutic drugs can be delivered to specific locations in the body with the aid of external magnetic fields, whilst minimizing their side effects in healthy cells or tissues..$^{2-5}$

MRI is widely used in modern clinical medicine as a diagnostic tool, and enables noninvasive and threedimensional visualization of biological phenomena in living organisms with high spatial and temporal resolution. ${ }^{6,7}$ As a consequence, considerable attention has been paid to multifunctional magnetic nanoparticles as potentially attractive stimuli-responsive MRI contrast agents, ${ }^{8,9}$ because their magnetism can be modulated by their dispersion state. ${ }^{10}$ However, sometimes the contrast difference between the biological tissues under investigation is trivial due to poor cellular uptake and failure to provide a high contrast signal. ${ }^{11}$ Therefore, a contrast agent with efficient targeting ability and cellular internalization ability is needed to provide higher contrast and information-rich images for disease detection.

Solid lipid nanoparticles, developed in the early 1990s, are composed of physiologically compatible lipid components which decrease the risk of acute and chronic toxicity. Compared with traditional drug carriers, solid lipid nanoparticles combine the advantages of polymeric nanoparticles and emulsions for drug delivery, including having low toxicity, good biocompatibility, and tumor targeting ability. ${ }^{12}$ Prolonged presence of the contrast agent at the target site is the primary requisite of any effective delivery system used for tumor imaging and diagnosis. In this regard, the effects of coating solid lipid nanoparticles with hydrophilic and flexible macromolecules such as poly(ethylene glycol) (PEG) to achieve a longer circulation time has been investigated. ${ }^{13-15}$ Moreover, for enhancing solid lipid nanoparticles as an imaging probe, it has been necessary to modify the surfaces of solid lipid nanoparticles with a ligand to facilitate selective targeting of cancer cells more efficiently without loss of affinity and specificity. Human epidermal growth factor receptor 2 (HER2, also known as ErbB2, c-erbB2 or HER2/ neu), plays an essential role in proliferation and antiapoptosis mechanisms in HER2-positive breast cancer. ${ }^{16}$ LTVSPWY, a homing peptide which was identified using a biopanning procedure, has been reported to be able to deliver an antisense oligonucleotide selectively to HER2-positive tumor cells by conjugation with the oligonucleotide. ${ }^{17}$

Herein, we report the use of a lipid-modified magnetic nanoparticle as a model carrier to improve the sensitivity and selectivity of $\mathrm{Fe}_{3} \mathrm{O}_{4}$-based MRI contrast agents.
To achieve this, we synthesized LTVSPWY-modified PEGylated chitosan (LTVSPWY-PEG-CS), and then prepared and characterized LTVSPWY-PEG-CS-modified magnetic nanoparticles. Their average diameter, zeta potential, and surface morphology were investigated in detail. The tumor targeting ability of the modified magnetic nanoparticles when used as an MRI contrast agent was studied in vitro in a coculture system containing SKOV-3 cells which overexpress the cell surface marker HER-2 and A549 cells which are HER2-negative, and in vivo in an SKOV-3 tumor cellbearing nude mouse model.

\section{Materials and methods}

Chitosan with a molecular weight of $18.0 \mathrm{kDa}$ was prepared by enzymatic degradation of chitosan $(95 \%$ deacetylate, molecular weight $450 \mathrm{kDa}$ ) sourced from Yuhuan Marine Biochemistry Co, Ltd, Zhejiang, China, as in our previous work. ${ }^{18} \mathrm{~N}, \mathrm{~N}^{\prime}$-disuccinimidyl carbonate (DSC) was purchased from Bio Basic Inc, Amherst NY. Di-tert-butyl dicarbonate [(Boc $\left.)_{2} \mathrm{O}\right]$, 1-ethyl-3-(3-dimethylaminopropyl) carbodiimide (EDC), and monostearin were purchased from Shanghai Medpep Co, Ltd, Shanghai, China. Oleic acid was purchased from Shuanglin Co, Ltd, Hangzhou, China. Trypsin and RPMI 1640 medium were purchased from Gibco BRL, Gaithersburg, MD. Fetal bovine serum was obtained from Hangzhou Sijiqing Biology Engineering Materials Co, Ltd, Hangzhou, China. Iron II, III oxide powder $\left(\mathrm{Fe}_{3} \mathrm{O}_{4},<50 \mathrm{~nm}\right)$ and 3-(4,5-dimethylthiazol-2-yl)-2,5-diphenyltetrazolium bromide (MTT) were purchased from Sigma Chemical Co, St Louis, MO. Rhodamine B isothiocyanate (RITC) was also purchased from Sigma. Hoechst 33342 was obtained from Acros Organics, Fair Lawn, NJ. PKH67 fluorescent cell linker was sourced from Sigma. 1, $1^{\prime}$-dioctadecyl-3,3,3', $\mathbf{3}^{\prime}$ tetramethyl indotricarbocyanine iodide (DiR) was purchased from Invitrogen, Grand Island, NY. All other reagents were analytical or chromatographic grade.

\section{Synthesis of LTVSPWY-PEG-CS copolymers}

Briefly, $10 \mathrm{mg}$ of LTVSPWY peptide was dissolved in dry dimethylformamide, and 3.1 $\mu \mathrm{L}(\mathrm{BOC})_{2} \mathrm{O}$ was added into the solution. The reaction was carried out for 24 hours at $40^{\circ} \mathrm{C}$ to protect the amine group in the LTVSPWY peptide. Next, $19.8 \mathrm{mg}$ of EDC and $20.8 \mathrm{mg}$ of $\mathrm{NH}_{2}-\mathrm{PEG}_{-} \mathrm{NH}_{2}$ were added, followed by stirring at $40^{\circ} \mathrm{C}$ for 24 hours. After that, the reaction solution was reacted with $\mathrm{N}, \mathrm{N}^{\prime}$-disuccinimidyl carbonate $\left(\mathrm{NH}_{2}-\mathrm{PEG}-\mathrm{NH}_{2}: \mathrm{DSC} 1: 1, \mathrm{~mol} / \mathrm{mol}\right)$ for 12 hours at $40^{\circ} \mathrm{C}$ to obtain a succinimidyl t-Boc-LTVSPWY-PEG$\mathrm{NH}_{2}$ solution. 
To obtain LTVSPWY-PEG-CS, chitosan was added into the reaction solution (chitosan:succinimidyl t-Boc-RGDPEG-NH $\mathrm{NH}_{2} 1: 1, \mathrm{~mol} / \mathrm{mol}$ ) and the reaction was allowed to continue at $40^{\circ} \mathrm{C}$ for 24 hours. To stop protection of the LTVSPWY peptide by (BOC) $)_{2} \mathrm{O}, 3 \mathrm{M} \mathrm{HCl}$ was added and allowed to react for 2 hours. The $\mathrm{pH}$ of the reaction solution was then adjusted to 7.0 using $3 \mathrm{M} \mathrm{NaOH}$. Finally, the reaction solution was dialyzed using a dialysis membrane (molecular weight cutoff $14 \mathrm{kDa}$; Spectrum Laboratories, Laguna Hills, CA) against distilled water for 24 hours, followed by lyophilization.

To obtain PEG-CS as a control, $200 \mathrm{mg}$ of chitosan were dissolved in $30 \mathrm{~mL}$ of distilled water. Then, $55 \mathrm{mg}$ of mPEG2000 with an aldehyde side group (PEG:chitosan $1: 1, \mathrm{~mol} / \mathrm{mol}$ ) was added into the chitosan-SA solution. The solution was stirred overnight at room temperature and then dialyzed against distilled water using a dialysis membrane (molecular weight cutoff $7 \mathrm{kDa}$, Spectrum Laboratories) for 24 hours. The final product was lyophilized.

\section{Preparation of magnetic nanoparticles}

Magnetic nanoparticles were prepared using the solvent diffusion method. Briefly, $5 \mathrm{mg}$ of $\mathrm{Fe}_{3} \mathrm{O}_{4}$ were added into $47 \mathrm{~mL}$ of $0.1 \%$ Poloxamer 188 in deionized water solution followed by sonication for 30 minutes using a probe sonicator (600 W, Sonicator JY92-II DN, Zhejiang, China) to form a dispersion of magnetic nanoparticles. To stabilize the nanoparticles, $1 \mathrm{~mL}$ of oleic acid in ethanol solution $(5 \mathrm{mg} / \mathrm{mL})$ was added and sonicated for 5 minutes. Next, $35 \mathrm{mg}$ of monostearin was dissolved in $2 \mathrm{~mL}$ of dimethyl sulfoxide at $70^{\circ} \mathrm{C}$ and immediately injected into the aqueous dispersion of magnetic nanoparticles in an ultrasound waterbath at $70^{\circ} \mathrm{C}$. The pre-emulsion was then cooled down to room temperature until magnetic nanoparticles were obtained.

To modify the magnetic nanoparticles further, an aqueous solution of LTVSPWY-PEG-CS (100 $\mu \mathrm{g} / \mathrm{mL})$ was added dropwise into the magnetic nanoparticle solution obtained under sonication using an ultrasound waterbath for 5 minutes to form LTVSPWY-PEG-CS-modified magnetic nanoparticles. As a control, PEG-CS or chitosan-modified magnetic nanoparticles were prepared as described above.

Characteristics of magnetic nanoparticles 'H NMR analysis of LTVSPWY-modified PEGylated chitosan

${ }^{1} \mathrm{H}$ NMR spectra were used to analyze the synthesized LTVSPWY-PEG-CS. LTVSPWY, PEG2000, chitosan, PEG-CS, and LTVSPWY-PEG-CS were dissolved in $\mathrm{D}_{2} \mathrm{O}$, and measured using an NMR spectrometer (AC-80, Bruker Biospin, Germany).

\section{Determination of particle size, zeta potential, and TEM}

The particle size and zeta potential of the magnetic nanoparticles were measured by dynamic light scattering using a Zetasizer (3000HS, Malvern Instruments Ltd, Worcestershire, UK). Morphological examination of the magnetic nanoparticles was performed using transmission electron microscopy (TEM, JEOL JEM-1230, Tokyo, Japan). The samples were stained with $2 \%(\mathrm{w} / \mathrm{v})$ phosphotungstic acid and placed on copper grids with films for viewing.

\section{Cell culture}

A SKOV-3 human ovarian carcinoma cell line and an A549 human lung carcinoma (alveolar type 2) cell line were obtained from the Cell Resource Center of the China Science Academy. The cells were cultured in RMPI 1640 medium at $37^{\circ} \mathrm{C}$ with $5 \% \mathrm{CO}_{2}$ under fully humidified conditions. All media were supplemented with $10 \%$ (v/v) fetal bovine serum, penicillin $100 \mathrm{U} / \mathrm{mL}$, and streptomycin $100 \mathrm{U} / \mathrm{mL}$. The cells were subcultured regularly using trypsin-ethylenediamine tetra-acetic acid.

\section{Cytotoxicity assay in vitro}

A comparison of cytotoxicity was performed on the test cells with in vitro proliferation using the MTT method. ${ }^{19}$ Briefly, SKOV-3 and A549 cells were plated in 96-well plates at a density of $1 \times 10^{4}$ cells/well in $200 \mu \mathrm{L}$ of complete medium, and incubated for 24 hours to allow the cells to attach. The cells were then exposed to serial concentrations of magnetic nanoparticles at $37^{\circ} \mathrm{C}$ for 48 hours. At the end of incubation, $20 \mu \mathrm{L}$ of the MTT solution was added, with incubation at $37^{\circ} \mathrm{C}$ for another 4 hours, and the medium was then replaced with $100 \mu \mathrm{L}$ of dimethyl sulfoxide to dissolve the MTT formazan crystals. The plates were shaken for 10 minutes and absorbance was measured at $570 \mathrm{~nm}$ using a microplate reader (BioRad, Model 680, Hercules, CA).

\section{Competitive cellular uptake in vitro}

Before incubation with the magnetic nanoparticles, the A549 cells were stained using a PKH67 fluorescent cell linker kit (Sigma), with the fluorescent cells becoming incorporated into the cell membrane with no modification of biological activity, ${ }^{20}$ following the manufacturer's protocol with some modification. Briefly, the cells were resuspended 
in $200 \mu \mathrm{L}$ diluent $\mathrm{C}$, and then $200 \mu \mathrm{L}$ of PHK67 dye ( $4 \mu \mathrm{M})$ was added, followed by incubation for 10 minutes at room temperature. To stop the staining reaction, $1 \mathrm{~mL}$ of serum was added and incubated for 2 minutes, followed by centrifugation at $400 \times \mathrm{g}$ for 10 minutes. The cell pellet was washed twice more with $10 \mathrm{~mL}$ of complete medium to ensure removal of unbound dye and resuspended to the desired concentration.

The PKH67-labeled HER2-negative A549 cells were then cocultured with the HER2-overexpressing SKOV-3 cells ${ }^{21}$ in the same well of a 24-well plate and incubated for 24 hours to allow attachment. The cells were then incubated with RITC-labeled magnetic nanoparticles $(2: 1, \mathrm{~mol} / \mathrm{mol})$ in growth medium for 12 hours. After washing the cells with phosphate-buffered solution three times, cellular uptake was observed using fluorescence microscopy (Olympus DP70, Melville, NY).

\section{Biodistribution of magnetic nanoparticles in vivo}

Far-red or near-infrared light (spectral range 650-900 nm) provides a "clear" window for in vivo optical imaging because it is separated from the major absorption peaks of blood and water. ${ }^{22}$ After optical calculations, we estimated that use of near-infrared-emitting DiR would improve tumor imaging sensitivity by at least ten-fold. DiR-loaded magnetic nanoparticles were prepared as described earlier, with some modifications. Briefly, a mixture of DiR and monostearin in dimethyl sulfoxide was injected into the aqueous dispersion of magnetic nanoparticles.

Tumor xenograft models were established by subcutaneous injection of approximately $5 \times 10^{6} \mathrm{SKOV}-3$ cells in
$100 \mu \mathrm{L}$ of serum-free RMPI 1640 medium into the flank of male $\mathrm{BALB} / \mathrm{C}+\mathrm{nu} / \mathrm{F} 1$ nude mice. The mice were then subjected to imaging studies when their tumors had reached an acceptable size. After tail vein injection of DiR-loaded magnetic nanoparticles (with $\mathrm{Fe}_{3} \mathrm{O}_{4}$, oleic acid, monostearin, chitosan, and DiR concentrations of 50, 50, 350, 50, and $200 \mu \mathrm{g} / \mathrm{mL}$, respectively), the mice were anesthetized and imaged at multiple time points $(1,3,6,12,24,48,72$, and 96 hours) using Maestro in vivo imaging system (CRI Inc, Woburn, MA). The tunable filter was automatically stepped up in $10 \mathrm{~nm}$ increments from $580 \mathrm{~nm}$ to $700 \mathrm{~nm}$ while the camera captured images at each wavelength with constant exposure. Overall acquisition time was about 0.4 seconds. For ex vivo imaging, the tissues were subjected to fluorescence imaging using the Spectral system immediately after the tumors and organs were harvested.

\section{Statistical analysis}

The data are expressed as the mean of three separate experiments. The statistical significance of the differences were determined using the Student's $t$-test for each paired experiment. A $P$ value $<0.05$ was considered to be significant in all cases.

\section{Results and discussion Synthesis and characterization of LTVSPWY-PEG-CS}

As shown in Figure 1, t-Boc-LTVSPWY-PEG-NH $\mathrm{N}_{2}$ was prepared by chemical reaction between the - $\mathrm{COOH}$ of LTVSPWY (its amino terminus preprotected by $(\mathrm{Boc})_{2} \mathrm{O}$ ) and the $-\mathrm{NH}_{2}$ of $\mathrm{NH}_{2}-\mathrm{PEG}-\mathrm{NH}_{2}$ in the presence of EDC. t-Boc-LTVSPWYPEG-CS was synthesized by conjugating the remaining amino

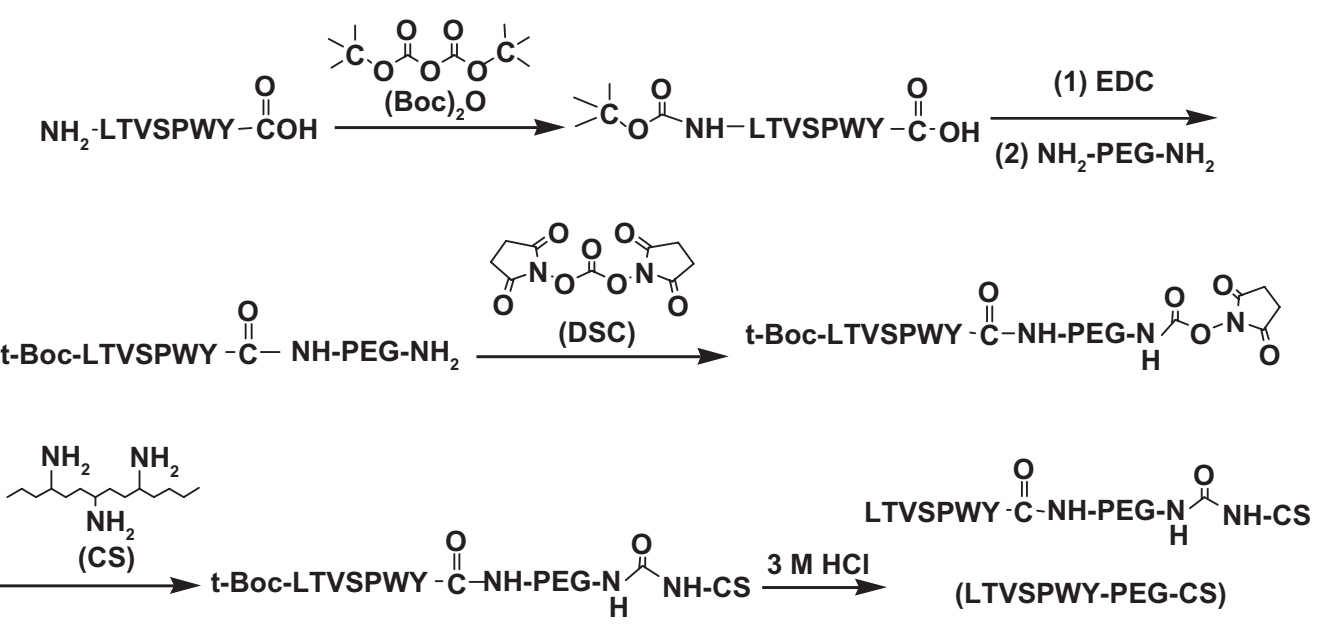

Figure I Synthetic scheme of LTVSPWY-PEG-CS.

Abbreviations: PEG, poly(ethylene glycol); CS, chitosan. 
groups of $\mathrm{NH}_{2}-\mathrm{PEG}-\mathrm{NH}_{2}$ and the primary amino groups of chitosan in the presence of DSC, which has been recognized as a versatile reagent for active synthesis of esters. ${ }^{23}$ In addition, PEG-CS was formed by reaction between the amino groups of chitosan and the aldehyde group of PEG. The chemical structure of the LTVSPWY-PEG-CS obtained was confirmed by ${ }^{1} \mathrm{H}$ NMR spectroscopy, and is shown in Figure 2. The peaks of the methylene protons in the ethylene glycol units $\left(\mathrm{CH}_{2} \mathrm{CH}_{2} \mathrm{O}\right)$ were at $3.58 \mathrm{ppm}$, and the benzene protons in the tryptophan and tyrosine units were at $8.32 \mathrm{ppm}$.

\section{Characteristics of magnetic nanoparticles}

As previous reported, oleic acid is used as a dispersant to obtain a stable aqueous dispersion of $\mathrm{Fe}_{3} \mathrm{O}_{4}$ nanoparticles. ${ }^{24}$ The oleic acid adsorbed onto the surface of $\mathrm{Fe}_{3} \mathrm{O}_{4}$ nanoparticles was then favored for the coating of solid lipid material monostearin by the solvent diffusion method due to the compatibility between oleic acid and monostearin. Furthermore, LTVSPWY-PEG-CS-modified magnetic nanoparticles were formed successfully by the charge interaction between LTVSPWY-PEG-CS with its positive charge and the magnetic nanoparticles with their negative charge. The average diameter and zeta potential of the magnetic nanoparticles are shown in Table 1. Modification with LTVSPWY-PEG-CS, PEG-CS, or chitosan increased the particles significantly. However, there was no significant difference in particle size between the three types of modified magnetic nanoparticles. With further modification of LTVSPWY-PEG-CS, the zeta potential of the magnetic nanoparticles as well as the PEG-CS

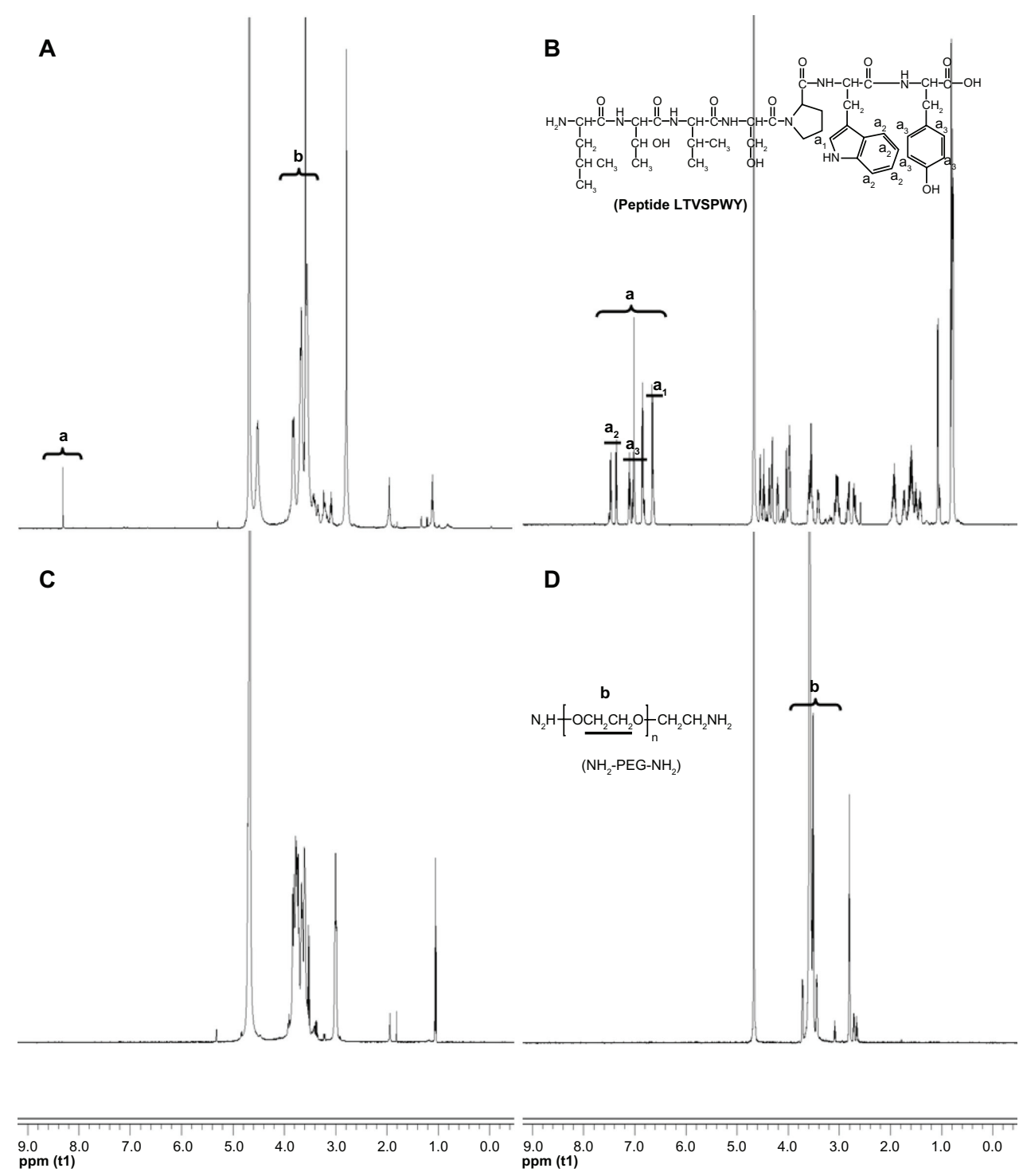

Figure 2 'H NMR spectra of LTVSPWY-PEG-CS (A), peptide LTVSPWY (B), CS (C) and $\mathrm{NH}_{2}-\mathrm{PEG}-\mathrm{NH}_{2}$ (D). The important peaks are pointed out. Abbreviations: PEG, poly(ethylene glycol); CS, chitosan. 
Table I Size and zeta potential of magnetic nanoparticles

\begin{tabular}{lllc}
\hline Sample & $\begin{array}{l}\text { Dn } \\
(\mathbf{n m})\end{array}$ & $\begin{array}{l}\text { PI } \\
(-)\end{array}$ & $\begin{array}{l}\text { Zeta potential } \\
(\mathbf{m V})\end{array}$ \\
\hline Non-modified MNPs & $156.0 \pm 11.5$ & $0.19 \pm 0.03$ & $-10.4 \pm 3.5$ \\
LTVSPWY-PEG-CS & $267.3 \pm 23.4$ & $0.55 \pm 0.14$ & $30.5 \pm 7.0$ \\
modified MNPs & & & \\
$\begin{array}{l}\text { PEG-CS-modified } \\
\text { MNPs }\end{array}$ & $275.0 \pm 59.4$ & $0.48 \pm 0.11$ & $22.1 \pm 0.8$ \\
CS-modified MNPs & $278.3 \pm 15.6$ & $0.40 \pm 0.09$ & $23.1 \pm 1.9$ \\
\hline
\end{tabular}

Note: Data represent the mean \pm standard deviation $(n=3)$. Abbreviations: MNPs, magnetic nanoparticles; PI, polydispersity index; dn, average diameter of magnetic nanoparticles; PEG, poly(ethylene glycol); CS, chitosan.

and chitosan-modified nanoparticles was reversed in the range of $-10.4 \pm 3.7 \mathrm{mV}$ to $30.5 \pm 7.0 \mathrm{mV}$. This charge reversal indicates that the LTVSPWY-PEG-CS-modified magnetic nanoparticles had been prepared successfully. Figure 3 shows the size distribution and zeta potential of the LTVSPWY-PEG-CS-modified magnetic nanoparticles obtained by dynamic light scattering, suggesting that they had a narrow size distribution.

TEM is a powerful tool for detecting particle size and surface morphology in nanoparticles. The spherical morphology of our magnetic nanoparticles was confirmed by TEM (Figure 4). It was found that the particle size observed under TEM was smaller than that obtained by dynamic light scattering, which may be due to shrinkage of the inner core of the magnetic nanoparticles during the drying process needed when preparing samples for TEM.

\section{In vitro cytotoxicity assay}

The cytotoxic effects of the magnetic nanoparticles against SKOV-3 and A549 cells was evaluated using the MTT test. Variations in cell viability according to nanoparticle concentration are shown in Figure 5. Regardless of the increasing magnetic nanoparticle concentration, the viability of SKOV-3 and A549 cells remained above $80 \%$, indicating that these nanoparticles would have low toxicity when used as carriers for cancer diagnostics.

\section{Competitive cellular uptake in vitro}

Using a coculture system containing SKOV-3 and A549 cells, the competitive cellular uptake of magnetic nanoparticles was investigated to determine the targeting ability of LTVSPWY-PEG-CS-modified magnetic nanoparticles toward SKOV-3 cells in vitro. The competitive cellular uptake was observed by fluorescence microscopy after the cells were incubated with LTVSPWY-PEG-CS-modified magnetic nanoparticles, PEG-CS-modified magnetic nanoparticles, or chitosan-modified magnetic nanoparticles for 12 hours. Figure 6 shows the fluorescence images of the effects of RITC-labeled magnetic nanoparticles in a cocultured system containing SKOV-3 and A549 cells.

Figure 6A shows clearly that there was a significant difference in uptake of LTVSPWY-PEG-CS-modified magnetic nanoparticles between SKOV-3 and A549 cells in the coculture system. Uptake of LTVSPWY-PEG-CSmodified magnetic nanoparticles by SKOV-3 cells was more efficient than by A549 cells, due to endocytosis mediated by the LTVSPWY homing peptide. However, the PEG-CSmodified magnetic nanoparticles showed similar uptake between SKOV-3 and A549 cells (Figure 6B), as did the chitosan-modified magnetic nanoparticles (Figure 6C). The competitive cellular uptake data confirm strong and specific binding of LTVSPWY-PEG-CS-modified magnetic nanoparticles with SKOV-3 cells, due to the abundant presence
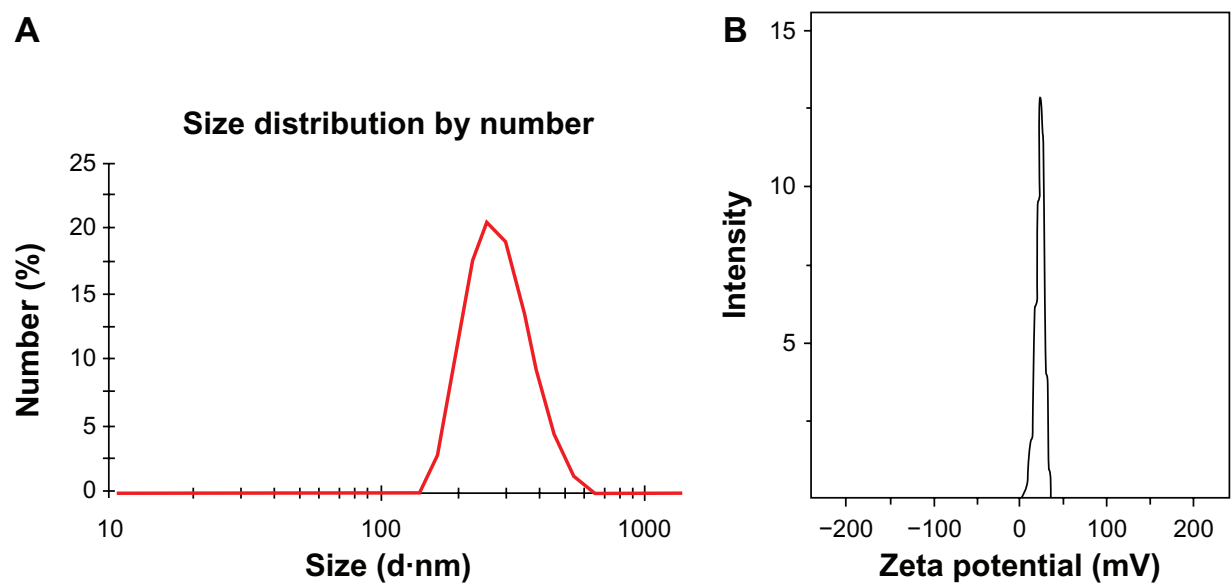

Figure 3 Characteristics of magnetic nanoparticles. Size distribution (A) and zeta potential (B) of LTVSPWY-PEG-CS-modified magnetic nanoparticles obtained by dynamic light scattering.

Abbreviations: PEG, poly(ethylene glycol); CS, chitosan. 

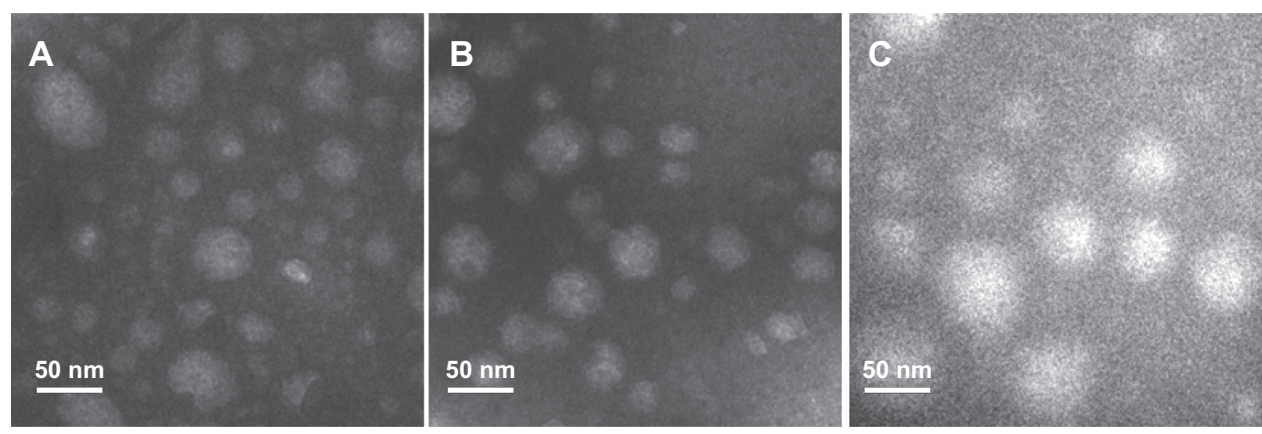

Figure 4 Transmission electron microscopic images of magnetic nanoparticles. (A) LTVSPWY-PEG-CS-modified magnetic nanoparticles, (B) PEG-CS-modified magnetic nanoparticles, and (C) CS-modified magnetic nanoparticles.

Note: The bar is $50 \mathrm{~nm}$.

Abbreviations: PEG, poly(ethylene glycol); CS, chitosan.
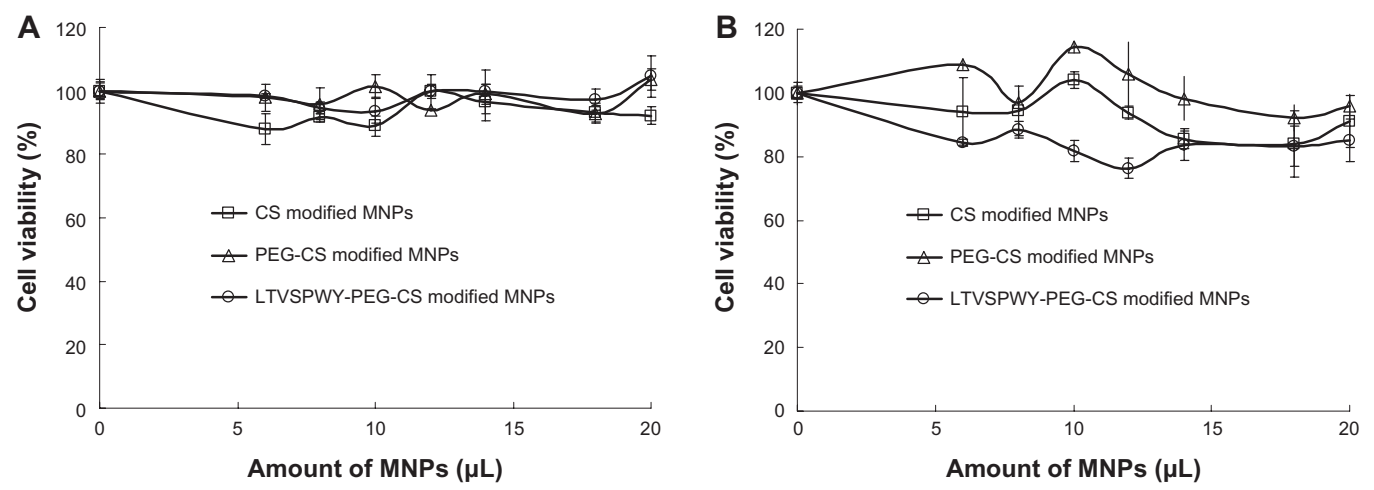

Figure 5 Cytotoxicity of magnetic nanoparticles against A549 (A) and SKOV-3 (B) cells. The magnetic nanoparticles prepared consisted of $50 \mu \mathrm{g} / \mathrm{mL} \mathrm{Fe}{ }_{3} \mathrm{O}_{4}, 50 \mu \mathrm{g} / \mathrm{mL}$ oleic acid, $350 \mu \mathrm{g} / \mathrm{mL}$ monostearin, and $50 \mu \mathrm{g} / \mathrm{mL}$ chitosan.

Note: Data represent the mean \pm standard deviation $(n=3)$.

Abbreviations: PEG, poly(ethylene glycol); CS, chitosan.

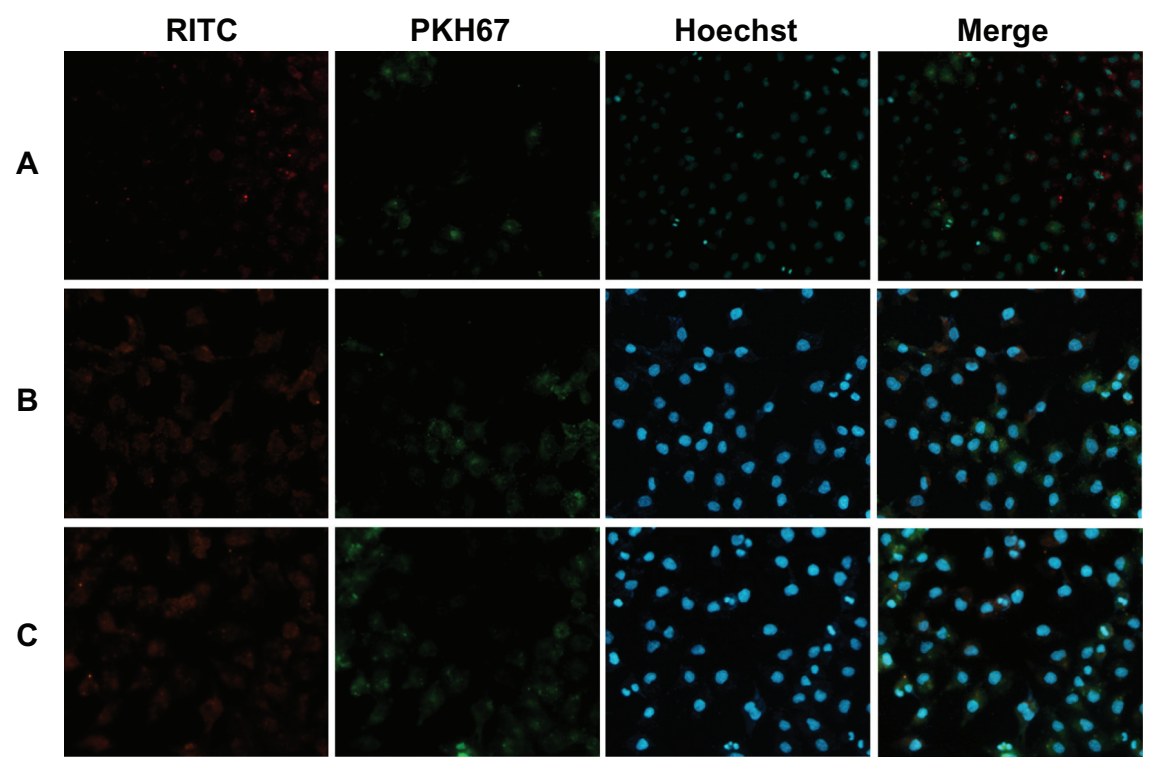

Figure 6 Fluorescence images of competitive cellular uptake of RITC-labeled magnetic nanoparticles for I2 hours. A549 cells (HER2-negative, cytoplasmic membrane-labeled with PKH67 fluorescent linker, green) cocultured with HER2-overexpressing SKOV-3 cells were incubated with RITC-labeled magnetic nanoparticles (red). (A) LTVSPWYPEG-CS-modified magnetic nanoparticles, (B) PEG-CS-modified magnetic nanoparticles, and (C) chitosan-modified magnetic nanoparticles.

Note: The cells were all stained with Hoechst 33342.

Abbreviations: RITC, rhodamine B isothiocyanate; PEG, poly(ethylene glycol); CS, chitosan. 


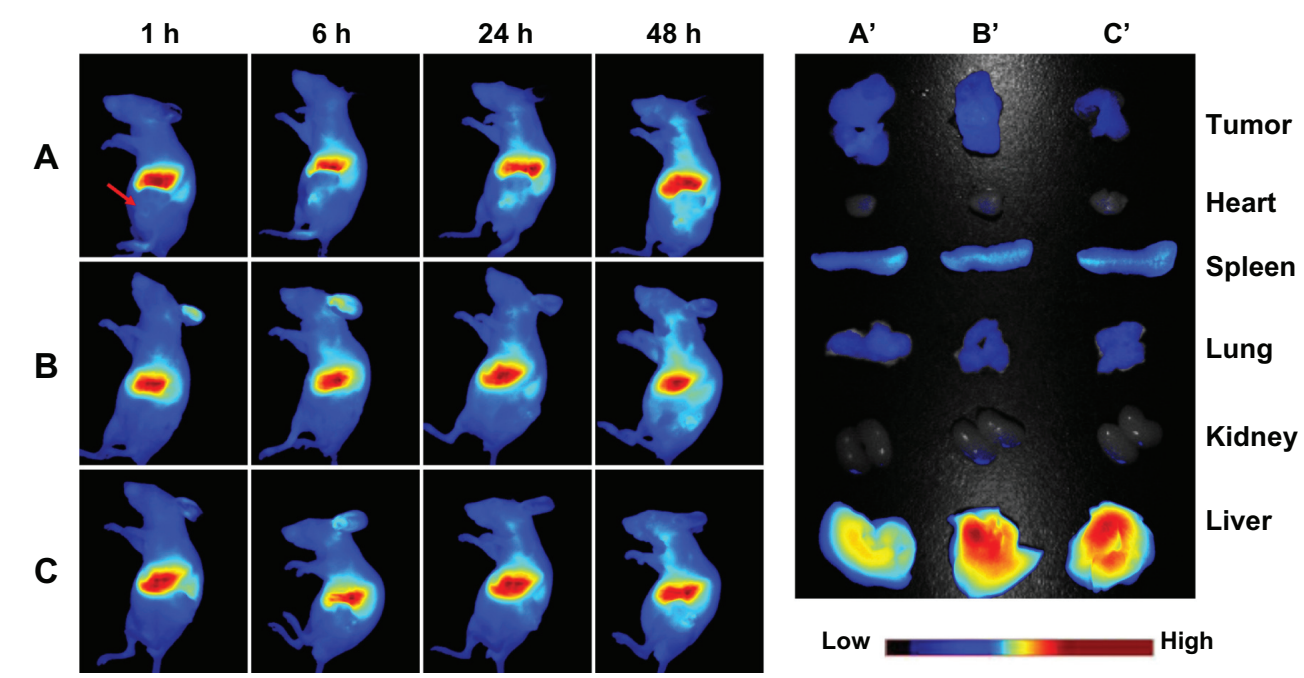

Figure 7 In vivo and ex vivo biodistribution of DiR-loaded magnetic nanoparticles after intravenous tail injection in tumor-bearing nude mice. The fluorescent images of in vivo tumor-bearing nude mice (A-B) and ex vivo image of major organs ( $\left.\mathbf{A}^{\prime}-\mathbf{C}^{\prime}\right)$ using DiR probes with three different magnetic nanoparticles, ie, (A and $\left.\mathbf{A}^{\prime}\right)$ LTVSPWY-PEG-CSmodified magnetic nanoparticles, (B and B') PEG-CS-modified magnetic nanoparticles, and (C and C') chitosan-modified magnetic nanoparticles.

Notes: The images were spectrally resolved. The tumor area is emphasized by a red arrow.

Abbreviations: PEG, poly(ethylene glycol); CS, chitosan.

of HER-2, a cell surface marker that is highly expressed by SKOV-3 cells. ${ }^{21}$ These results confirm that LTVSPWYPEG-CS-modified magnetic nanoparticles retain the binding activity and specificity of the LTVSPWY peptide, and may be used as an active targeting carrier via LTVSPWY peptide mediation.

\section{Biodistribution of magnetic nanoparticles in vivo}

The in vivo behavior of the magnetic nanoparticles was then assessed following tail vein injection in a nude mouse model of a SKOV-3 xenograft in the left flank. The fluorescent images obtained at 1, 6, 24, and 48 hours after injection of DiR-loaded magnetic nanoparticles are shown in Figure 7. The uptake and retention of magnetic nanoparticles took place primarily in the liver, with little accumulation in tumor tissue. The maximum fluorescence signal is achieved at 48 hours after injection, and the tumor tissue is clearly delineated. Similar to the results obtained for cell uptake in vitro, more active accumulation of LTVSPWY-PEG-CSmodified magnetic nanoparticles was observed in a short time (one hour) within the area of the tumor (Figure 7A), in comparison with the PEG-CS-modified and chitosanmodified magnetic nanoparticles (Figure 7B and C). For PEG-CS-modified magnetic nanoparticles, the circulation time was increased, leading to slower accumulation of the magnetic nanoparticles in the tumors compared with the chitosan-modified magnetic nanoparticles.
Observation of the organs 96 hours after injection and dissection of the animals confirmed similar accumulation of LTVSPWY-PEG-CS-modified and PEG-CS-modified magnetic nanoparticles in the tumors. However, accumulation of LTVSPWY-PEG-CS-modified magnetic nanoparticles in the liver was reduced compared with that of the PEG-CSmodified and chitosan-modified ones. The biodistribution results obtained in this study indicate that modification by PEG, which prevents protein binding, can prolong the blood circulation time of the magnetic nanoparticles in vivo. More importantly, active tumor targeting using a tumor-specific ligand is much faster and more efficient than passive targeting based on tumor permeation, uptake, and retention. ${ }^{25}$ Therefore, LTVSPWY-PEG-CS-modified magnetic nanoparticles can be delivered to tumors by both passive and active targeting mechanisms under in vivo conditions.

\section{Conclusion}

In summary, LTVSPWY-PEG-CS-modified magnetic nanoparticles were successfully prepared using the solvent diffusion method. With modification by the LTVSPWY homing peptide, these magnetic nanoparticles could be taken up selectively by HER2-overexpressing SKOV-3 cells when cocultured with HER2-negative A549 cells, and with low toxicity. Treatment using LTVSPWY-PEG-CS-modified DiR-loaded magnetic nanoparticles enabled the tumors to be identified and diagnosed more rapidly and efficiently in vivo. Peptide-based nanoprobes may open up new opportunities 
for the development of novel molecular imaging probes in the early detection and diagnosis of cancer.

\section{Acknowledgment}

We are grateful for the financial support of the National Nature Science Foundation of China under contract 81171334 and Medical and health research funding schemes of Zhejiang Province under contract 2012KYB098.

\section{Disclosure}

The authors report no conflicts of interest in this work.

\section{References}

1. Mahmoudi M, Simchi A, Imani M, et al. A new approach for the in vitro identification of the cytotoxicity of superparamagnetic iron oxide nanoparticles. Colloids Surf B. 2010;75(1):300-309.

2. Polyak B, Friedman G. Magnetic targeting for site-specific drug delivery: applications and clinical potential. Expert Opin Drug Deliv. 2009;6(1):53-70.

3. Lubbe AS, Alexiou C, Bergemann C. Clinical applications of magnetic drug targeting. J Surg Res. 2001;95(2):200-206.

4. Gupta AK, Gupta M. Synthesis and surface engineering of iron oxide nanoparticles for biomedical applications. Biomaterials. 2005;26(18): 3995-4021.

5. Sunderland CJ, Steiert M, Talmadge JE, Derfus AM, Barry SE. Targeted nanoparticles for detecting and treating cancer. Drug Dev Res. 2006;67(1):70-93.

6. Okada S, Mizukami S, Kikuchi K. Switchable MRI contrast agents based on morphological changes of $\mathrm{pH}$-responsive polymers. Bioorgan Med Chem. 2012;20(2):769-774.

7. Kaur S, Venktaraman G, Jain M, Senapati S, Garg PK, Batra SK. Recent trends in antibody-based oncologic imaging. Cancer Lett. 2012;315(2): 97-111.

8. Briley-Saebo K, Bjørnerud A, Grant D, Ahlstrom H, Berg T, Kindberg GM. Hepatic cellular distribution and degradation of iron oxide nanoparticles following single intravenous injection in rats: implications of magnetic resonance imaging. Cell Tissue Res. 2004;316(3): 315-323.

9. Singh A, Dilnawaz F, Mewar S, Sharma U, Jagannathan NR, Sahoo SK. Composite polymeric magnetic nanoparticles for co-delivery of hydrophobic and hydrophilic anticancer drugs and MRI imaging for cancer therapy. ACS Appl Mater Interfaces. 2011;3(3):842-856.

10. Tanaka K, Kitamura N, Morita M, Inubushi T, Chujo Y. Assembly system of direct modified superparamagnetic iron oxide nanoparticles for target-specific MRI contrast agents. Bioorg Med Chem Lett. 2008; 18(20):5463-5465.
11. Corot C, Robert P, Idee JM, Port M. Recent advances in iron oxide nanocrystal technology for medical imaging. Adv Drug Deliv Rev. 2006;58(14):1471-1504.

12. Sarmento B, Mazzaglia D, Bonferoni MC, Neto AP, Monteiro MC, Seabra V. Effect of chitosan coating in overcoming the phagocytosis of insulin loaded solid lipid nanoparticles by mononuclear phagocyte system. Carbohydr Polym. 2011;84(3):919-925.

13. Gao JQ, Eto Y, Yoshioka Y, et al. Effective tumor targeted gene transfer using PEGylated adenovirus vector via systemic administration. $J$ Control Release. 2007;122(1):102-110.

14. Inada Y, Furukawa M, Sasaki H, et al. Biomedical and biotechnological applications of PEG- and PM-modified proteins. Trends Biotechnol. 1995;13(3):86-91.

15. Hong JW, Park JH, Huh KM, Chung H, Kwon IC, Jeong SY. PEGylated polyethylenimine for in vivo local gene delivery based on lipiodolized emulsion system. J Control Release. 2004;99(1):167-176.

16. Tai W, Mahato R, Cheng K. The role of HER 2 in cancer therapy and targeted drug delivery. J Control Release. 2010;146(3):264-275.

17. Shadidi M, Sioud M. Identification of novel carrier peptides for the specific delivery of therapeutics into cancer cells. FASEB $J$. 2003;17(2):256-258.

18. Hu FQ, Ren GF, Yuan H, Du YZ, Zeng S. Shell cross-linked stearic acid grafted chitosan oligosaccharide self-aggregated micelles for controlled release of paclitaxel. Colloids Surf B. 2005;50(2):97-103.

19. Mosmann T. Rapid colorimetric assay for cellular growth and survival: application to proliferation and cytotoxicity assays. J Immunol Methods. 1983;65(1-2):55-63.

20. Martina MS, Wilhelm C, Lesieur S. The effect of magnetic targeting on the uptake of magnetic-fluid-loaded liposomes by human prostatic adenocarcinoma cells. Biomaterials. 2008;29(30):4137-4145.

21. Lewis Phillips GD, Li G, Dugger DL, et al. Targeting HER2-positive breast cancer with trastuzumab-DM1, an antibody-cytotoxic drug conjugate. Cancer Res. 2008;68(22):9280-9290.

22. Tziachristos V, Bremer C, Weissleder R. Fluorescence imaging with near-infrared light: new technological advances that enable in vivo molecular imaging. Eur Radiol. 2003;13(1):195-208.

23. Boden N, Bushby RJ, Liu Q, et al. N, N'-Disuccinimidyl carbonate as a coupling agent in the synthesis of thiophospholipids used for anchoring biomembranes to gold surfaces. Tetrahedron. 1998;54(38): 11537-11548

24. Ying XY, Du YZ, Hong LH, Yuan H, Hu FQ. Magnetic lipid nanoparticles loading doxorubicin for intracellular delivery: preparation and characteristics. J Magn Magn Mater. 2011;323(8):1088-1093.

25. Gao X, Cui Y, Levenson RM, Chung LWK, Nie S. In vivo cancer targeting and imaging with semiconductor quantum dots. Nat Biotechnol. 2004;22(8):969-976.
International Journal of Nanomedicine

\section{Publish your work in this journal}

The International Journal of Nanomedicine is an international, peerreviewed journal focusing on the application of nanotechnology in diagnostics, therapeutics, and drug delivery systems throughou the biomedical field. This journal is indexed on PubMed Central, MedLine, CAS, SciSearch ${ }^{\circledR}$, Current Contents ${ }^{\circledR} /$ Clinical Medicine,

\section{Dovepress}

Journal Citation Reports/Science Edition, EMBase, Scopus and the Elsevier Bibliographic databases. The manuscript management system is completely online and includes a very quick and fair peer-review system, which is all easy to use. Visit http://www.dovepress.com/ testimonials.php to read real quotes from published authors. 